\title{
Natural Heritage Vegetation Database for West Virginia
}

\author{
James Vanderhorst, Elizabeth Byers \& Brian Streets
}

\begin{abstract}
The West Virginia Division of Natural Resources, Natural Heritage Program maintains a database of vegetation plots sampled across the state, the Natural Heritage Vegetation Database for West Virginia (GIVD ID NA-US-013). Data includes information on vegetation structure, plant species composition, and environment in plots of specified area and location. The purpose of this database is to facilitate classification and characterization of natural and semi-natural vegetation in the state to serve as a tool for conservation of biological diversity in all kingdoms.
\end{abstract}

Keywords: conservation; vegetation plot.

GIVD Database ID: NA-US-013

Last update: $2012-01-27$

\section{Natural Heritage Vegetation Database for West Virginia}

Scope: Our database includes vegetation plot data collected from throughout the state of West Virginia. Plot data is limited to natural and seminatural vegetation, and emphasis is placed on high quality stands. Plots must sample a specific area and meet minimum standards for locational, environmental, and floristic data. Floristic lists must be reasonably complete for the defined area, with percentage cover estimated for each plant taxon.

Status: completed and continuing

Period: 1963-2012

Database manager(s): James Vanderhorst (James.P.Vanderhorst@wv.gov); Elizabeth Byers (Elizabeth.A.Byers@wv.gov); Brian Streets (Brian.P.Streets@wv.gov)

Owner: West Virginia Division of Natural Resources

Natural Heritage Program

PO Box 67

Elkins, West Virginia 26241

Web address: [NA]

Availability: according to a specific agreement

Database format(s): MS Access

Publication: [NA]

Plot type(s): normal plots; time series

Non-overlapping plots: 3,896

Total plot observations: 3,905

Online upload: no

Online search: no

Countries: US: $100.0 \%$

Export format(s): MS Access, Excel, PDF, CSV file, plain text file

Forest: [NA] — Non-forest: [NA]

Guilds: all vascular plants: $100 \%$; bryophytes (terricolous or aquatic): $22 \%$; lichens (terricolous or aquatic): $22 \%$; algae (terricolous or aquatic): $1 \%$ Environmental data: altitude: $100 \%$; slope aspect: $73 \%$; slope inclination: $50 \%$; microrelief: $6 \%$; soil depth: $50 \%$; surface cover other than plants (open soil, litter, bare rock etc.): $60 \%$; soil pH: $40 \%$; land use categories: $19 \%$

Performance measure(s): cover: $100 \%$; measurements like diameter or height of trees: $80 \%$

Geographic localisation: GPS coordinates (precision $25 \mathrm{~m}$ or less): 53\%; point coordinates less precise than GPS, up to $1 \mathrm{~km}$ : $46 \%$; political units or only on a coarser scale $(>10 \mathrm{~km}): 1 \%$

Sampling periods: 1960-1969: 1.0\%; 1980-1989: 2.0\%; 1990-1999: 36.0\%; 2000-2009: 52.0\%; 2010-2019: 10.0\% Information as of 2012-07-12; further details and future updates available from http://www.givd.info/ID/NA-US-013

James Vanderhorst*(James.P.Vanderhorst@wv.gov), Elizabeth Byers (Elizabeth.A.Byers@wv.gov), Brian Streets

(Brian.P.Streets@wv.gov)

West Virginia Division of Natual Resources, Natural Hertitage Program, PO Box 67, 26241 Elkins, UNITED STATES

*Corresponding author 